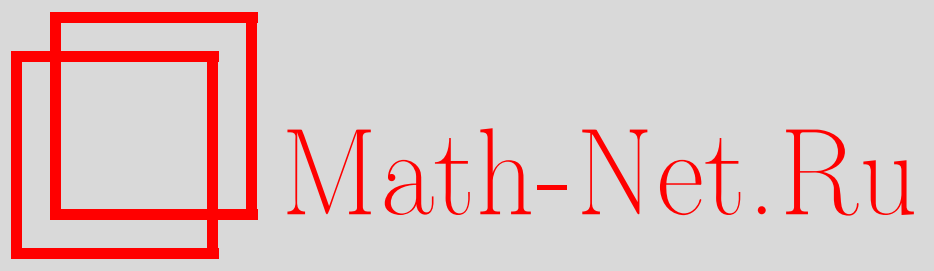

С. Мирзаеи, Г. Наярбаши, М. А. Фасихи, Ф. Мирмасоуди, Запутанность многочастичных фермионных когерентных состояний псевдоэрмитовых гамильтонианов, TMФ, 2018, том 196, номер 1, 99-116

DOI: https://doi.org/10.4213/tmf9467

Использование Общероссийского математического портала Math-Net.Ru подразумевает, что вы прочитали и согласны с пользовательским соглашением http://www . mathnet.ru/rus/agreement

Параметры загрузки:

IP: 54.80 .73 .141

26 апреля 2023 г., 16:04:57

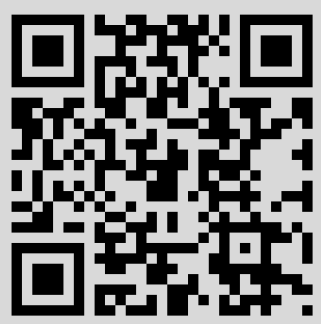




\title{
ЗАПУТАННОСТЬ МНОГОЧАСТИЧНЫХ ФЕРМИОННЫХ КОГЕРЕНТНЫХ СОСТОЯНИЙ ПСЕВДОЭРМИТОВЫХ ГАМИЛЬТОНИАНОВ
}

\begin{abstract}
Изучается запутанность мультикубитных фермионных псевдоэрмитовых когерентных состояний, которые описываются антикоммутативными грассмановыми числами. Путем интегрирования по тензорным произведениям этих состояний с использованием подходящих грассмановых весовых функций введены псевдоэрмитовы версии таких хорошо известных максимально запутанных чистых состояний, как состояния Белла, состояния Гринбергера-Хорна-Цайлингера, состояния Вернера и бисепарабельные состояния. В качестве иллюстрации предложенный подход применен к тензорному произведению двух- и трехкубитных псевдоэрмитовых состояний. Для количественной характеристики запутанности таких состояний применяются меры запутанности, заданные соответственно функцией конкуренции и средней энтропией.
\end{abstract}

Ключевые слова: псевдоэрмитов, запутанность, псевдофермионные когерентные состояния, псевдосостояния Белла, псевдосостояния Гринбергера-Хорна-Цайлингера, псевдосостояния Вернера.

DOI: https://doi.org/10.4213/tmf9467

\section{1. ВВЕДЕНИЕ}

Научная непротиворечивость квантовой теории информации в последнее время возросла благодаря получению нескольких выдающихся результатов. Наиболее важным из них было то, что явления запутанности квантовых состояний [1] получили надежную теоретическую формулировку и экспериментальную проверку с помощью некоторых тестов [2]-[9]. Фактически запутанность является наиболее

${ }^{*}$ Department of Physics, Faculty of Sciences, Sahand University of Technology, Tabriz, Iran. E-mail: s.mirzaei@sut.ac.ir

${ }^{\dagger}$ Department of Physics, University of Mohaghegh Ardabili, Ardabil, Iran. E-mail: najarbashi@uma.ac.ir

${ }^{\ddagger}$ Department of Physics, Azarbayjan Shahid Madani University, Tabriz, Iran. E-mail fasihi@azaruniv.edu 
интересной и в то же время странной особенностью квантовой физики. Идея запутывания возникает из очевидного конфликта между принципом суперпозиции и несепарабельностью соответствующих квантовых состояний. Запутывание имеет место, когда состояние двух или более подсистем составной квантовой системы нельзя представить как произведение чистых локальных состояний подсистем. Другими словами, запутанное состояние можно использовать для направления удаленной частицы в одно из множества состояний с определенной вероятностью.

Недавние исследования в области теоретической физики и квантовой оптики показали важность когерентных состояний. Их можно применять для кодирования квантовой информации в непрерывных переменных [10]. В то время как запутанность бозонных когерентных $s u(2)$ - и $s u(1,1)$-состояний как неортогональных состояний, играющих важную роль в квантовой криптографии и обработке квантовой информации, широко изучалась в научной литературе [11]-[17], свойства запутанности многочастичных фермионных когерентных состояний остаются нерешенной проблемой квантовой теории информации даже с теоретической точки зрения [18]-[22]. Фермионные когерентные состояния определяются как собственные состояния оператора уничтожения, отвечающие грассмановым собственным значениям [23]-[26].

С другой стороны, в последнее десятилетие возрос интерес к неэрмитовым гамильтонианам с действительными спектрами [25], [27]-[34]. В работах [28], [29] Бендер с соавторами, рассматривая результаты различных численных расчетов, нашли некоторые примеры одномерных неэрмитовых гамильтонианов, которые обладают действительным спектром. Поскольку такие гамильтонианы инвариантны относительно обращения времени и четности, их спектральные свойства связаны с их РТсимметрией. В работах [33]-[35] понятие псевдоэрмитовости было введено как возможный альтернативный инструмент изучения неэрмитовых операторов, допускающих действительный спектр. Кроме того, псевдоэрмитовы гамильтонианы использовались при изучении некоторых специфических эффектов в физике конденсированных сред [36], [37].

Недавно в работе [20] было проведено исследование запутанности грассмановых когерентных состояний для многочастичных $n$-уровневых эрмитовых систем. Для этого рассматривалось тензорное произведение $|\theta\rangle=|0\rangle-\theta|1\rangle$ одномодовых фермионных когерентных состояний (т. е. состояний $\left|\theta_{1}\right\rangle\left|\theta_{2}\right\rangle$ ), которое представлялось в терминах стандартного базиса $|0\rangle,|1\rangle$ и антикоммутирующих грассмановых чисел $\left(\theta_{i} \theta_{j}=-\theta_{j} \theta_{i}\right)$. Такое правило оправданно в контексте квантовой теории поля, где, например, тензорное произведение двух одночастичных состояний является двухчастичным состоянием и т. д. Авторы работы [20] нашли стандартные максимально запутанные состояния Белла, состояния Гринбергера-Хорна-Цайлингера (далее GHZ-состояния) и состояния Вернера (W-состояния) путем интегрирования по тензорному произведению двух-, трех- и мультимодовых фермионных когерентных состояний с подходящими весовыми функциями.

Целью настоящей работы является распространение указанного метода на псевдоэрмитовы системы. Для них вместо стандартного базиса мы используем два набора базисных состояний $\left\{\left|\psi_{0}\right\rangle,\left|\psi_{1}\right\rangle\right\}$ и $\left\{\left|\phi_{0}\right\rangle,\left|\phi_{1}\right\rangle\right\}$, которые являются собственными для операторов $H$ и $H^{\dagger}$ соответственно. В результате мы имеем два возможных фермионных псеводоэрмитовых когерентных состояния $\left(\right.$ ФЭКС) $|\theta\rangle=\left|\psi_{0}\right\rangle-\theta\left|\psi_{1}\right\rangle$ и $|\tilde{\theta}\rangle=\left|\phi_{0}\right\rangle-\theta\left|\phi_{1}\right\rangle$. 
Если заданы чистые коррелированные или некоррелированные системы $A$ и $B$, то состояние $|\psi\rangle_{A B}$ двухчастичной составной системы есть сумма тензорных произведений $|i\rangle_{A} \otimes|j\rangle_{B}$ :

$$
|\psi\rangle_{A B}=\sum_{i, j=0,1} a_{i j}|i\rangle_{A} \otimes|j\rangle_{B} .
$$

При этом мы использовали тот факт, что, как принято в стандартной квантовой теории информации, блоховский вектор (или кубит) $|\psi\rangle=a|0\rangle+|1\rangle$ разложен по стандартному базису $\{|0\rangle,|1\rangle\}$. Этот базис используется для построения таких запутанных состояний, как:

$$
\begin{aligned}
& \text { состояния Белла } \frac{|00\rangle \pm|11\rangle}{\sqrt{2}}, \frac{|01\rangle \pm|10\rangle}{\sqrt{2}}, \\
& \text { GHZ-состояния } \frac{|000\rangle \pm|111\rangle}{\sqrt{2}}, \\
& \mathrm{~W} \text {-состояния } \frac{|001\rangle+|010\rangle+|011\rangle}{\sqrt{3}} \text { и т. д. }
\end{aligned}
$$

Эти состояния и их расширения являются полезными инструментами при обработке квантовой информации в том смысле, что они используются в различных задачах, включая квантовую телепортацию [38], квантовую криптографию [39], удаленное приготовление квантовых состояний [40] и квантовую связь [41]. Тесно связанное с этими задачами расширение с математической точки зрения означает восстановление запутанных состояний со следующей заменой:

- сумма $\sum(\cdot)$ заменяется на интеграл $\int(\cdot) d \theta_{1} d \theta_{2}$,

- коэффициенты $a_{i j}$ заменяются на грассманову весовую функцию $w\left(\theta_{1}, \theta_{2}\right)$,

- базис $|i\rangle_{A}|j\rangle_{B}$ заменяется на грассмановы когерентные состояния $\left|\theta_{1}\right\rangle\left|\theta_{2}\right\rangle$.

В этом случае расширение состояния (1.1) подразумевает псевдоэрмитовость и содержит мультимодовые фермионные когерентные состояния, которые включают в себя грассмановы (антикоммутирующие) переменные интегрирования. Применяя интегральный подход псевдоквантовой механики, мы готовим почву для введения методов функционального интегрирования по полям для спиновых систем, рассматривающихся в контексте теории многих тел [42].

Настоящая статья состоит из двух основных частей. Первая из них посвящена построению различных семейств псевдоэрмитовых версий известных максимально запутаннных чистых состояний, таких как состояния Белла, GHZ-состояния и W-состояния, а также псевдобисепарабельные состояния, путем интегрирования по тензорному произведению ФПЭКС двух и трех однокубитных псевдоэрмитовых систем при надлежащем выборе грассмановой весовой функции. В разделе 2 мы даем краткое введение в псевдоэрмитову квантовую механику. В разделе 3 , используя результаты для обобщенных грассмановых псевдоэрмитовых состояний [26], мы представляем ФПЭКС как особый случай обобщенных грассмановых псевдоэрмитовых состояний двухуровневой системы. В разделе 4 мы строим псевдоэрмитову версию состояний Белла, GHZ-состояний и W-состояний.

Во второй части, а именно в разделе 5, мы используем функцию конкуренции, чтобы количественно описать запутанность псевдосостояний Белла, и среднюю энтропию, чтобы количественно описать запутанность GHZ-состояний и W-состояний, а затем сравниваем наши результаты со случаем эрмитовых максимально запутанных чистых состояний. Заключительные замечания содержатся в разделе 6. 


\section{2. ПСЕВДОЭРМИТОВЫ ГАМИЛЬТОНИАНЫ И БИОРТОНОРМИРОВАННЫЙ БАЗИС СОБСТВЕННЫХ СОСТОЯНИЙ}

Уравнение Шредингера с комплексными потенциалами, но при этом с действительным спектром интенсивно исследовалось различными методами. Пионерские статьи [27]-[34] инициировали исследование РТ-симметричных систем, а затем Мустафазаде ввел более общий класс псевдоэрмитовых моделей [33], [34]. Следуя его подходу, предположим, что $H: \mathcal{H} \rightarrow \mathcal{H}$ есть линейный оператор, действующий в гильбертовом пространстве $\mathcal{H}$, и $\eta: \mathcal{H} \rightarrow \mathcal{H}$ является линейным эрмитовым автоморфизмом (обратимым преобразованием). Тогда оператор, $\eta$-псевдоэрмитовосопряженный к $H$, задается как $H^{\sharp}=\eta^{-1} H^{\dagger} \eta$. Говорят, что оператор $H$ является псевдоэрмитовым по отношению к автоморфизму $\eta$ или просто $\eta$-псевдоэрмитовым, если $H^{\sharp}=H$. Собственные значения псевдоэрмитова гамильтониана $H$ либо действительны, либо возникают комплексно-сопряженными парами. При этом в невырожденном случае имеет место равенство

$$
H^{\dagger}=\eta H \eta^{-1}
$$

Для диагонализуемого оператора $H$ с дискретным спектром существует полный биортонормированный собственный базис $\left\{\left|\psi_{i}\right\rangle,\left|\phi_{i}\right\rangle\right\}$, такой что

$$
\begin{gathered}
H\left|\psi_{i}\right\rangle=E_{i}\left|\psi_{i}\right\rangle, \quad H^{\dagger}\left|\phi_{i}\right\rangle=\bar{E}_{i}\left|\phi_{i}\right\rangle, \\
\left\langle\phi_{i} \mid \psi_{j}\right\rangle=\delta_{i j}, \quad \sum_{i}\left|\psi_{i}\right\rangle\left\langle\phi_{i}\left|=\sum_{i}\right| \phi_{i}\right\rangle\left\langle\psi_{i}\right|=I .
\end{gathered}
$$

Для заданного псевдоэрмитова оператора $H$ существует бесконечно много автоморфизмов $\eta$, для которых выполнено равенство (2.1). Однако их можно записать в терминах полного биортонормированного базиса оператора $H$. В невырожденном случае преобразование $\eta$ и его обратное, для которых выполнено равенство (2.1), имеют вид

$$
\begin{array}{ll}
\eta=\sum_{i}\left|\phi_{i}\right\rangle\left\langle\phi_{i}\right|, \quad & \eta^{-1}=\sum_{i}\left|\psi_{i}\right\rangle\left\langle\psi_{i}\right|, \\
\left|\phi_{i}\right\rangle=\eta\left|\psi_{i}\right\rangle, & \left|\psi_{i}\right\rangle=\eta^{-1}\left|\phi_{i}\right\rangle .
\end{array}
$$

Всюду в настоящей статье мы полагаем, что псевдогамильтониан $H$ и, следовательно, преобразование $\eta$ действуют в двумерном гильбертовом пространстве.

\section{3. ФПЭКС}

3.1. Грассмановы переменные. Обсуждение основных свойств грассмановых переменных можно найти в работах [43]-[46]. Для наших целей здесь мы дадим краткий обзор свойств алгебры, порожденной переменными $\theta_{1}, \ldots, \theta_{n}$, которые по определению удовлетворяют следующим свойствам:

$$
\theta_{i}^{2}=0, \quad \theta_{i} \theta_{j}=-\theta_{j} \theta_{i}, \quad j=1, \ldots, n .
$$

Аналогичные правила также выполняются для переменных $\theta^{\dagger}=\bar{\theta}$, эрмитово-сопряженных к $\theta$ :

$$
\bar{\theta}_{i}^{2}=0, \quad \bar{\theta}_{i} \bar{\theta}_{j}=-\bar{\theta}_{j} \bar{\theta}_{i}, \quad i, j=1, \ldots, n .
$$


Кроме того, аналогично $\theta_{i} \bar{\theta}_{j}=-\bar{\theta}_{j} \theta_{i}$. Любая линейная комбинация переменных $\theta_{i}$ с комплексными коэффициентами называется грассмановым числом. Другими словами, разложение Тейлора для грассмановой функции записывается как

$$
g\left(\theta_{1}, \ldots, \theta_{n}\right)=c_{0}+\sum_{i=1} c_{i} \theta_{i}+\sum_{i, j} c_{i, j} \theta_{i} \theta_{j}+\cdots,
$$

где $c_{0}, c_{i}, c_{i, j}$ - комплексные числа. Например, $e^{\theta_{1} \theta_{2}}=1+\theta_{1} \theta_{2}$. Интегрирование и дифференцирование по грассмановым переменным задается по правилам Березина:

$$
\begin{gathered}
\int d \theta f(\theta)=\frac{\partial f(\theta)}{\partial \theta}, \\
\int d \theta=0, \quad \int d \theta \theta=1, \quad \int d \bar{\theta}=0, \quad \int d \bar{\theta} \bar{\theta}=1, \\
\frac{\partial}{\partial \theta} \theta=1, \quad \frac{\partial}{\partial \theta} 1=0, \quad \frac{\partial}{\partial \bar{\theta}} \bar{\theta}=1, \quad \frac{\partial}{\partial \bar{\theta}} 1=0, \quad \frac{\partial^{2}}{\partial \theta^{2}}=0, \quad \frac{\partial^{2}}{\partial \bar{\theta}^{2}}=0 .
\end{gathered}
$$

Чтобы вычислить интеграл от проивольной функции по алгебре Грассмана, требуется выполнение следующих соотношений:

$$
\begin{array}{rlrl}
\theta d \bar{\theta}=-d \bar{\theta} \theta, \quad \bar{\theta} d \theta & =-d \theta \bar{\theta}, & \theta d \theta & =-d \theta \theta, \quad \bar{\theta} d \bar{\theta}=-d \bar{\theta} \bar{\theta}, \\
d \theta d \bar{\theta} & =-d \bar{\theta} d \theta, \quad \theta \bar{\theta}=-\bar{\theta} \theta .
\end{array}
$$

3.2. Когерентные состояния. Следуя работам [25], [26], можно построить ФПЭКС двухуровневого псевдоэрмитова гамильтониана. Приведем основные результаты. Учитывая биортогональную природу псевдоэрмитовых систем, зададим две пары операторов рождения и уничтожения, соответствующих биортогональным собственным состояниям $\left|\psi_{i}\right\rangle,\left|\phi_{i}\right\rangle$, как

$$
\begin{aligned}
b & :=\left|\psi_{0}\right\rangle\left\langle\phi_{1}\right|, & \tilde{b} & =\eta b \eta^{-1}=\left|\phi_{1}\right\rangle\left\langle\psi_{0}\right|, \\
b^{\sharp} & :=\eta^{-1} b^{\dagger} \eta=\left|\psi_{1}\right\rangle\left\langle\phi_{0}\right|, & \tilde{b}^{\sharp \prime} & =\eta^{\prime-1} b^{\dagger} \eta=\left|\phi_{0}\right\rangle\left\langle\psi_{1}\right|,
\end{aligned}
$$

где $\eta^{\prime-1}=\eta$ и $b b^{\sharp}+b^{\sharp} b=I$. Тогда два семейства когерентных состояний двухуровневой псевдоэрмитовой грассмановой системы можно построить в терминах $\left|\psi_{k}\right\rangle$ и $\left|\phi_{k}\right\rangle$. Соответствующие состояниям $\left|\psi_{k}\right\rangle,\left|\phi_{k}\right\rangle \Phi П Э \mathrm{KC}$, которые мы обозначим как $|\theta\rangle,|\tilde{\theta}\rangle$, по определению являются собственными состояниями операторов уничтожения $b$ и $\tilde{b}$,

$$
b|\theta\rangle=\theta|\theta\rangle, \quad \tilde{b}|\tilde{\theta}\rangle=\theta|\tilde{\theta}\rangle,
$$

и с точностью до нормировки имеют вид

$$
|\theta\rangle=\left|\psi_{0}\right\rangle-\theta\left|\psi_{1}\right\rangle, \quad|\tilde{\theta}\rangle=\left|\phi_{0}\right\rangle-\theta\left|\phi_{1}\right\rangle
$$

Можно использовать явный вид двух семейств ФПЭКС и свойство биортогональности псевдоэрмитовой системы для нахождения возможных интегралов от $|\theta\rangle$ и $|\tilde{\theta}\rangle$, т. е. $|\theta\rangle\langle\tilde{\theta}|$ и $|\tilde{\theta}\rangle\langle\theta|$, по мере $d \bar{\theta} d \theta w(\theta, \bar{\theta})$. Это приводит к тождеству

$$
\int d \bar{\theta} d \theta w(\theta, \bar{\theta})|\theta\rangle\left\langle\tilde{\theta}\left|=\int d \bar{\theta} d \theta w(\theta, \bar{\theta})\right| \tilde{\theta}\right\rangle\langle\theta|=I
$$


где $w(\theta, \bar{\theta})=1+\theta \bar{\theta}$. Тождество (3.8) называется бисоотношением полноты. Чтобы вычислить весовую функцию, мы налагаем на биортогональные собственные состояния $\left|\psi_{k}\right\rangle,\left|\phi_{k}\right\rangle(k=0,1)$ и грассмановы переменные $\theta, \bar{\theta}$ следующие требования квантования:

$$
\begin{aligned}
\theta\left|\psi_{k}\right\rangle & =(-1)^{k-1}\left|\psi_{k}\right\rangle \theta, & & \bar{\theta}\left\langle\psi_{k}\right|=(-1)^{k-1}\left\langle\psi_{k}\right| \bar{\theta}, \\
\theta\left\langle\psi_{k}\right| & =(-1)^{k-1}\left\langle\psi_{k}\right| \theta, & & \bar{\theta}\left|\psi_{k}\right\rangle=(-1)^{k-1}\left|\psi_{k}\right\rangle \bar{\theta}, \\
\theta\left|\phi_{k}\right\rangle & =(-1)^{k-1}\left|\phi_{k}\right\rangle \theta, & \bar{\theta}\left\langle\phi_{k}\right| & =(-1)^{k-1}\left\langle\phi_{k}\right| \bar{\theta}, \\
\theta\left\langle\phi_{k}\right| & =(-1)^{k-1}\left\langle\phi_{k}\right| \theta, & & \bar{\theta}\left|\phi_{k}\right\rangle=(-1)^{k-1}\left|\phi_{k}\right\rangle \bar{\theta} .
\end{aligned}
$$

Приведеное выше обсуждение демонстрирует, что ни интеграл $|\theta\rangle\langle\theta|$, ни интеграл $|\tilde{\theta}\rangle\langle\tilde{\theta}|$ по мере $d \bar{\theta} d \theta w(\theta, \bar{\theta})$ не являются нормированными:

$$
\int d \bar{\theta} d \theta w(\theta, \bar{\theta})|\theta\rangle\left\langle\theta\left|\neq I, \quad \int d \bar{\theta} d \theta w(\theta, \bar{\theta})\right| \tilde{\theta}\right\rangle\langle\tilde{\theta}| \neq I .
$$

Можно показать, что фермионные когерентные состояния (3.7) остаются когерентными при всех временах, если временная эволюция начальных состояний, задающаяся гамильтонианом, также есть собственное состояние понижающих операторов.

\section{4. МАКСИМАЛЬНО ЗАПУТАННЫЕ ПСЕВДОСОСТОЯНИЯ}

Рассмотрим фермионную систему, в которой частицы могут проходить по $n$-модовым каналам. С этой целью рассмотрим тензорное произведение $n$ одномодовых ФПЭКС, каждое из которых управляется псевдоэрмитовым гамильтонианом. Для простоты возьмем $n=2,3$, случай произвольного $n$ получается прямым обобщением. Введем псевдоэрмитову версию хорошо известных максимально запутанных чистых двух- и трехкубитных состояний, а именно состояний Белла и GHZ-состояний, Wсостояний соответственно [47], проинтегрировав по тензорному произведению ФПЭКС с подходящей грассмановой весовой функцией.

4.1. Псевдоэрмитовы состояния типа состояний Белла. Начнем с ненормированной псевдоэрмитовой версии стандартных состояний Белла

$$
\left|\Psi^{ \pm}\right\rangle=\frac{|01\rangle \pm|10\rangle}{\sqrt{2}}, \quad\left|\Phi^{ \pm}\right\rangle=\frac{|00\rangle \pm|11\rangle}{\sqrt{2}},
$$

т. е. с состояния

$$
\left|B_{1}^{-}\right\rangle=\left|\psi_{0}\right\rangle\left|\psi_{1}\right\rangle-\left|\psi_{1}\right\rangle\left|\psi_{0}\right\rangle
$$

Чтобы получить данное состояние, рассмотрим тензорное произведение двух одномодовых ФПЭКС с одинаковыми грассмановыми числами:

$$
|\theta\rangle|\theta\rangle=\left|\psi_{0}\right\rangle\left|\psi_{0}\right\rangle+\theta\left(\left|\psi_{0}\right\rangle\left|\psi_{1}\right\rangle-\left|\psi_{1}\right\rangle\left|\psi_{0}\right\rangle\right)
$$

Как отмечалось выше, этот метод становится понятным в контексте квантовой теории поля. Для получения выражения (4.3) мы воспользовались явной формулой (3.7) для $|\theta\rangle$. 
Следующим шагом является подбор походящей весовой функции $w(\theta)$, такой что интегрирование по грассмановым числам $\theta$ приводит к формуле (4.2). Для этого положим

$$
\int d \theta w(\theta)|\theta\rangle|\theta\rangle=\left|B_{1}^{-}\right\rangle
$$

если $w(\theta)=c_{0}+c_{1} \theta$, то (4.4) дает $c_{0}=1$ и $c_{1}=0$, и мы получаем подходящую для наших целей волновую функцию $w(\theta)=1$.

Рассматривая тензорные произведения $|\theta\rangle|\tilde{\theta}\rangle,|\tilde{\theta}\rangle|\theta\rangle$ и $|\tilde{\theta}\rangle|\tilde{\theta}\rangle$ при $w(\theta)=1$, можно построить другую форму псевдосостояний Белла:

$$
\left|B_{2}^{-}\right\rangle=\int d \theta|\theta\rangle|\tilde{\theta}\rangle=\left|\psi_{0}\right\rangle\left|\varphi_{1}\right\rangle-\left|\psi_{1}\right\rangle\left|\varphi_{0}\right\rangle
$$

До сих пор мы рассматривали тензорное произведение двух одномодовых ФПЭКС с одинаковыми грассмановыми числами и получили в результате псевдоэрмитову версию состояния $\left|\Psi^{-}\right\rangle$. Чтобы создать другое псевдосостояние Белла, нам требуется рассмотреть тензорное произведение ФПЭКС с различными грассмановыми числами:

$$
\left|\theta_{1}\right\rangle\left|\theta_{2}\right\rangle=\left|\psi_{0}\right\rangle\left|\psi_{0}\right\rangle+\theta_{2}\left|\psi_{0}\right\rangle\left|\psi_{1}\right\rangle-\theta_{1}\left|\psi_{1}\right\rangle\left|\psi_{0}\right\rangle+\theta_{1} \theta_{2}\left|\psi_{1}\right\rangle\left|\psi_{1}\right\rangle
$$

В этом случае общий вид весовой функции таков: $w\left(\theta_{1}, \theta_{2}\right)=c_{0}+c_{1} \theta_{1}+c_{2} \theta_{2}+c_{3} \theta_{1} \theta_{2}$. Теперь возникает задача найти такую $w\left(\theta_{1}, \theta_{2}\right)$, чтобы в дополнение к вышеупомянутому состоянию $\left|B_{i}^{-}\right\rangle$получить три других семейства псевдосостояний Белла. Обозначим их как $\left|B_{i}^{+}\right\rangle$и $\left|B_{i}^{\prime}{ }^{ \pm}\right\rangle$. Результат приведен в табл. 1. Например, псевдосостояние Белла $\left|\phi_{0}\right\rangle\left|\psi_{1}\right\rangle+\left|\phi_{1}\right\rangle\left|\psi_{0}\right\rangle$ получается, если взять тензорное произведение $\left|\tilde{\theta}_{1}\right\rangle\left|\theta_{2}\right\rangle$ c $w\left(\theta_{1}, \theta_{2}\right)=-\theta_{1}-\theta_{2}$.

ТАБлица 1. Ненормированные псевдосостояния Белла и соответствующие весовые функции.

\begin{tabular}{|c|c|c|c|}
\hline Состояние & ФПЭКС & Весовая функция & Псевдосостояние Белла \\
\hline$\left|B_{1}^{ \pm}\right\rangle$ & $\left|\theta_{1}\right\rangle\left|\theta_{2}\right\rangle$ & $-\left(\theta_{1} \pm \theta_{2}\right)$ & $\left|\psi_{0}\right\rangle\left|\psi_{1}\right\rangle \pm\left|\psi_{1}\right\rangle\left|\psi_{0}\right\rangle$ \\
$\left|B_{2}^{ \pm}\right\rangle$ & $\left|\theta_{1}\right\rangle\left|\tilde{\theta}_{2}\right\rangle$ & $-\left(\theta_{1} \pm \theta_{2}\right)$ & $\left|\psi_{0}\right\rangle\left|\phi_{1}\right\rangle \pm\left|\psi_{1}\right\rangle\left|\phi_{0}\right\rangle$ \\
$\left|B_{3}^{ \pm}\right\rangle$ & $\left|\tilde{\theta}_{1}\right\rangle\left|\theta_{2}\right\rangle$ & $-\left(\theta_{1} \pm \theta_{2}\right)$ & $\left|\phi_{0}\right\rangle\left|\psi_{1}\right\rangle \pm\left|\phi_{1}\right\rangle\left|\psi_{0}\right\rangle$ \\
$\left|B_{4}^{ \pm}\right\rangle$ & $\left|\tilde{\theta}_{1}\right\rangle\left|\tilde{\theta}_{2}\right\rangle$ & $-\left(\theta_{1} \pm \theta_{2}\right)$ & $\left|\phi_{0}\right\rangle\left|\phi_{1}\right\rangle \pm\left|\phi_{1}\right\rangle\left|\phi_{0}\right\rangle$ \\
\hline \hline$\left|B_{1}^{\prime \pm}\right\rangle$ & $\left|\theta_{1}\right\rangle\left|\theta_{2}\right\rangle$ & $-\left(\theta_{1} \theta_{2} \pm 1\right)$ & $\left|\psi_{0}\right\rangle\left|\psi_{0}\right\rangle \pm\left|\psi_{1}\right\rangle\left|\psi_{1}\right\rangle$ \\
$\left|B_{2}^{\prime \pm}\right\rangle$ & $\left|\theta_{1}\right\rangle\left|\tilde{\theta}_{2}\right\rangle$ & $-\left(\theta_{1} \theta_{2} \pm 1\right)$ & $\left|\psi_{0}\right\rangle\left|\phi_{0}\right\rangle \pm\left|\psi_{1}\right\rangle\left|\phi_{1}\right\rangle$ \\
$\left|B_{3}^{\prime \pm}\right\rangle$ & $\left|\tilde{\theta}_{1}\right\rangle\left|\theta_{2}\right\rangle$ & $-\left(\theta_{1} \theta_{2} \pm 1\right)$ & $\left|\phi_{0}\right\rangle\left|\psi_{0}\right\rangle \pm\left|\phi_{1}\right\rangle\left|\psi_{1}\right\rangle$ \\
$\left|B_{4}^{\prime \pm}\right\rangle$ & $\left|\tilde{\theta}_{1}\right\rangle\left|\tilde{\theta}_{2}\right\rangle$ & $-\left(\theta_{1} \theta_{2} \pm 1\right)$ & $\left|\phi_{0}\right\rangle\left|\phi_{0}\right\rangle \pm\left|\phi_{1}\right\rangle\left|\phi_{1}\right\rangle$ \\
\hline \hline$\left|B_{1}^{-}\right\rangle$ & $|\theta\rangle|\theta\rangle$ & 1 & $\left|\psi_{0}\right\rangle\left|\psi_{1}\right\rangle-\left|\psi_{1}\right\rangle\left|\psi_{0}\right\rangle$ \\
$\left|B_{2}^{-}\right\rangle$ & $|\theta\rangle|\tilde{\theta}\rangle$ & 1 & $\left|\psi_{0}\right\rangle\left|\phi_{1}\right\rangle-\left|\psi_{1}\right\rangle\left|\phi_{0}\right\rangle$ \\
$\left|B_{3}^{-}\right\rangle$ & $|\tilde{\theta}\rangle|\theta\rangle$ & 1 & $\left|\phi_{0}\right\rangle\left|\psi_{1}\right\rangle-\left|\phi_{1}\right\rangle\left|\psi_{0}\right\rangle$ \\
$\left|B_{4}^{-}\right\rangle$ & $|\tilde{\theta}\rangle|\tilde{\theta}\rangle$ & 1 & $\left|\phi_{0}\right\rangle\left|\phi_{1}\right\rangle-\left|\phi_{1}\right\rangle\left|\phi_{0}\right\rangle$ \\
\hline
\end{tabular}


4.2. Псевдоэрмитовы GHZ-состояния и W-состояния. Построим псевдоверсию GHZ-состояний и $\mathrm{W}$-состояний

$$
\left|G H Z^{ \pm}\right\rangle=\frac{|000\rangle \pm|111\rangle}{\sqrt{2}}, \quad|W\rangle=\frac{|100\rangle+|010\rangle+|001\rangle}{\sqrt{3}},
$$

которые широко известны в квантовой теории информации. Чтобы построить трехкубитное GHZ-псевдосостояние, нам необходимо рассмотреть тензорное произведение трех одномодовых ФПЭКС с различными грассмановыми числами, которое может иметь один из следующих восьми видов:

$$
\begin{array}{llll}
\left|\theta_{1}\right\rangle\left|\theta_{2}\right\rangle\left|\theta_{3}\right\rangle, & \left|\tilde{\theta}_{1}\right\rangle\left|\theta_{2}\right\rangle\left|\theta_{3}\right\rangle, & \left|\theta_{1}\right\rangle\left|\tilde{\theta}_{2}\right\rangle\left|\theta_{3}\right\rangle, & \left|\theta_{1}\right\rangle\left|\theta_{2}\right\rangle\left|\tilde{\theta}_{3}\right\rangle, \\
\left|\tilde{\theta}_{1}\right\rangle\left|\tilde{\theta}_{2}\right\rangle\left|\theta_{3}\right\rangle, & \left|\tilde{\theta}_{1}\right\rangle\left|\theta_{2}\right\rangle\left|\tilde{\theta}_{3}\right\rangle, & \left|\theta_{1}\right\rangle\left|\tilde{\theta}_{2}\right\rangle\left|\tilde{\theta}_{3}\right\rangle, & \left|\tilde{\theta}_{1}\right\rangle\left|\tilde{\theta}_{2}\right\rangle\left|\tilde{\theta}_{3}\right\rangle .
\end{array}
$$

В качестве примера рассмотрим состояния

$$
\left|G_{1}^{ \pm}\right\rangle=\int d \theta_{1} d \theta_{2} d \theta_{3} w^{ \pm}\left(\theta_{1}, \theta_{2}, \theta_{3}\right)\left|\theta_{1}\right\rangle\left|\theta_{2}\right\rangle\left|\theta_{3}\right\rangle=\left|\psi_{0}\right\rangle\left|\psi_{0}\right\rangle\left|\psi_{0}\right\rangle \pm\left|\psi_{1}\right\rangle\left|\psi_{1}\right\rangle\left|\psi_{1}\right\rangle,
$$

где весовые функции записываются как

$$
w^{ \pm}\left(\theta_{1}, \theta_{2}, \theta_{3}\right)=\theta_{3} \theta_{2} \theta_{1} \pm 1 \text {. }
$$

Нетрудно проверить, что эта функция является подходящей для каждого из состояний $\left|G_{i}^{ \pm}\right\rangle, i=1, \ldots, 8$. В результате мы получаем ненормированные GHZ-псевдосостояния, приведенные в табл. 2.

ТАБлицА 2. Ненормированные GHZ-псевдосостояния и соответствующие весовые функции.

\begin{tabular}{|c|c|c|c|}
\hline Состояние & ФПЭКС & Весовая функция & GHZ-псевдосостояние \\
\hline$\left|G_{1}^{ \pm}\right\rangle$ & $\left|\theta_{1}\right\rangle\left|\theta_{2}\right\rangle\left|\theta_{3}\right\rangle$ & $\theta_{3} \theta_{2} \theta_{1} \pm 1$ & $\left|\psi_{0}\right\rangle\left|\psi_{0}\right\rangle\left|\psi_{0}\right\rangle \pm\left|\psi_{1}\right\rangle\left|\psi_{1}\right\rangle\left|\psi_{1}\right\rangle$ \\
$\left|G_{2}^{ \pm}\right\rangle$ & $\left|\tilde{\theta}_{1}\right\rangle\left|\theta_{2}\right\rangle\left|\theta_{3}\right\rangle$ & $\theta_{3} \theta_{2} \theta_{1} \pm 1$ & $\left|\varphi_{0}\right\rangle\left|\psi_{0}\right\rangle\left|\psi_{0}\right\rangle \pm\left|\varphi_{1}\right\rangle\left|\psi_{1}\right\rangle\left|\psi_{1}\right\rangle$ \\
$\left|G_{3}^{ \pm}\right\rangle$ & $\left|\theta_{1}\right\rangle\left|\tilde{\theta}_{2}\right\rangle\left|\theta_{3}\right\rangle$ & $\theta_{3} \theta_{2} \theta_{1} \pm 1$ & $\left|\psi_{0}\right\rangle\left|\varphi_{0}\right\rangle\left|\psi_{0}\right\rangle \pm\left|\psi_{1}\right\rangle\left|\varphi_{1}\right\rangle\left|\psi_{1}\right\rangle$ \\
$\left|G_{4}^{ \pm}\right\rangle$ & $\left|\theta_{1}\right\rangle\left|\theta_{2}\right\rangle\left|\tilde{\theta}_{3}\right\rangle$ & $\theta_{3} \theta_{2} \theta_{1} \pm 1$ & $\left|\psi_{0}\right\rangle\left|\psi_{0}\right\rangle\left|\varphi_{0}\right\rangle \pm\left|\psi_{1}\right\rangle\left|\psi_{1}\right\rangle\left|\varphi_{1}\right\rangle$ \\
$\left|G_{5}^{ \pm}\right\rangle$ & $\left|\tilde{\theta}_{1}\right\rangle\left|\tilde{\theta}_{2}\right\rangle\left|\theta_{3}\right\rangle$ & $\theta_{3} \theta_{2} \theta_{1} \pm 1$ & $\left|\varphi_{0}\right\rangle\left|\varphi_{0}\right\rangle\left|\psi_{0}\right\rangle \pm\left|\varphi_{1}\right\rangle\left|\varphi_{1}\right\rangle\left|\psi_{1}\right\rangle$ \\
$\left|G_{6}^{ \pm}\right\rangle$ & $\left|\tilde{\theta}_{1}\right\rangle\left|\theta_{2}\right\rangle\left|\tilde{\theta}_{3}\right\rangle$ & $\theta_{3} \theta_{2} \theta_{1} \pm 1$ & $\left|\varphi_{0}\right\rangle\left|\psi_{0}\right\rangle\left|\varphi_{0}\right\rangle \pm\left|\varphi_{1}\right\rangle\left|\psi_{1}\right\rangle\left|\varphi_{1}\right\rangle$ \\
$\left|G_{7}^{ \pm}\right\rangle$ & $\left|\theta_{1}\right\rangle\left|\tilde{\theta}_{2}\right\rangle\left|\tilde{\theta}_{3}\right\rangle$ & $\theta_{3} \theta_{2} \theta_{1} \pm 1$ & $\left|\psi_{0}\right\rangle\left|\varphi_{0}\right\rangle\left|\varphi_{0}\right\rangle \pm\left|\psi_{0}\right\rangle\left|\varphi_{1}\right\rangle\left|\varphi_{0}\right\rangle$ \\
$\left|G_{8}^{ \pm}\right\rangle$ & $\left|\tilde{\theta}_{1}\right\rangle\left|\tilde{\theta}_{2}\right\rangle\left|\tilde{\theta}_{3}\right\rangle$ & $\theta_{3} \theta_{2} \theta_{1} \pm 1$ & $\left|\varphi_{0}\right\rangle\left|\varphi_{0}\right\rangle\left|\varphi_{0}\right\rangle \pm\left|\varphi_{1}\right\rangle\left|\varphi_{1}\right\rangle\left|\varphi_{1}\right\rangle$ \\
\hline
\end{tabular}

Чтобы построить W-псевдосостояния, необходимо использовать тензорное произведение ФПЭКС либо с тремя различными, либо с одинаковыми грассмановыми числами. Ниже мы представим по одному примеру состояний $\mathcal{W}$ и $\mathcal{W}^{\prime}$ для этих двух категорий.

Для тензорного произведения ФПЭКС с различными грассмановыми числами имеем

$$
\begin{aligned}
\left|\mathcal{W}_{1}\right\rangle & =\int d \theta_{1} d \theta_{2} d \theta_{3} w_{1}\left(\theta_{1}, \theta_{2}, \theta_{3}\right)\left|\theta_{1}\right\rangle\left|\theta_{2}\right\rangle\left|\theta_{3}\right\rangle= \\
& =\left|\psi_{0}\right\rangle\left|\psi_{0}\right\rangle\left|\psi_{1}\right\rangle+\left|\psi_{0}\right\rangle\left|\psi_{1}\right\rangle\left|\psi_{0}\right\rangle+\left|\psi_{1}\right\rangle\left|\psi_{0}\right\rangle\left|\psi_{0}\right\rangle
\end{aligned}
$$


где

$$
w\left(\theta_{1}, \theta_{2}, \theta_{3}\right)=\theta_{1} \theta_{2}+\theta_{1} \theta_{3}+\theta_{2} \theta_{3}
$$

Аналогично в случае совпадающих грассмановых чисел имеем

$$
\left|\mathcal{W}_{1}^{\prime}\right\rangle=\int d \theta w(\theta)|\theta\rangle|\theta\rangle|\theta\rangle=-\left|\psi_{0}\right\rangle\left|\psi_{0}\right\rangle\left|\psi_{1}\right\rangle+\left|\psi_{0}\right\rangle\left|\psi_{1}\right\rangle\left|\psi_{0}\right\rangle-\left|\psi_{1}\right\rangle\left|\psi_{0}\right\rangle\left|\psi_{0}\right\rangle
$$

где подходящей весовой функцией является $w^{\prime}(\theta)=1$.

Как видно из табл. 3, для заданного тензорного произведения $\left|\theta_{1}\right\rangle\left|\theta_{2}\right\rangle\left|\theta_{3}\right\rangle$ трех различных одномодовых ФПЭКС в зависимости от выбора весовой функции существуют восемь $\mathrm{W}$-псевдосостояний. Почеркнем, что, хотя мы строим состояния $\mathcal{W}^{\prime}$ в терминах $Ф$ ПКС с одинаковыми грассмановыми числами, можно попробовать получить тот же результат, используя различные грассмановы числа, что в свою очередь дает весовую функцию $w=-\theta_{1} \theta_{2}+\theta_{1} \theta_{3}-\theta_{2} \theta_{3}$.

ТАБлицА 3. Ненормированные $\mathrm{W}$-псевдосостояния и соответствующие весовые функции. Верхний индекс $(i)$ соответствует комбинациям символов $(+,+,+),(+,+,-), \ldots,(-,-,-)$, отвечающим весовым функциям $\theta_{1} \theta_{2}+$

\begin{tabular}{|c|c|c|c|}
\hline $\begin{array}{c}\text { Состо- } \\
\text { яние }\end{array}$ & $\Phi П Э К С$ & Весовая функция & W-псевдосостояние \\
\hline$\left|W_{1}^{(i)}\right\rangle$ & $\left|\theta_{1}\right\rangle\left|\theta_{2}\right\rangle\left|\theta_{3}\right\rangle$ & $\pm \theta_{1} \theta_{2} \pm \theta_{1} \theta_{3} \pm \theta_{2} \theta_{3}$ & $\pm\left|\psi_{0}\right\rangle\left|\psi_{0}\right\rangle\left|\psi_{1}\right\rangle \pm\left|\psi_{0}\right\rangle\left|\psi_{1}\right\rangle\left|\psi_{0}\right\rangle \pm\left|\psi_{1}\right\rangle\left|\psi_{0}\right\rangle\left|\psi_{0}\right\rangle$ \\
\hline$\left|W_{2}^{(i)}\right\rangle$ & $\left|\tilde{\theta}_{1}\right\rangle\left|\theta_{2}\right\rangle\left|\theta_{3}\right\rangle$ & $\pm \theta_{1} \theta_{2} \pm \theta_{1} \theta_{3} \pm \theta_{2} \theta_{3}$ & $\pm\left|\varphi_{0}\right\rangle\left|\psi_{0}\right\rangle\left|\psi_{1}\right\rangle \pm\left|\varphi_{0}\right\rangle\left|\psi_{1}\right\rangle\left|\psi_{0}\right\rangle \pm\left|\varphi_{1}\right\rangle\left|\psi_{0}\right\rangle\left|\psi_{0}\right\rangle$ \\
\hline$\left|W_{3}^{(i)}\right\rangle$ & $\left|\theta_{1}\right\rangle\left|\tilde{\theta}_{2}\right\rangle\left|\theta_{3}\right\rangle$ & $\pm \theta_{1} \theta_{2} \pm \theta_{1} \theta_{3} \pm \theta_{2} \theta_{3}$ & $\pm\left|\psi_{0}\right\rangle\left|\varphi_{0}\right\rangle\left|\psi_{1}\right\rangle \pm\left|\psi_{0}\right\rangle\left|\varphi_{1}\right\rangle\left|\psi_{0}\right\rangle \pm\left|\psi_{1}\right\rangle\left|\varphi_{0}\right\rangle\left|\psi_{0}\right\rangle$ \\
\hline$\left|W_{4}^{(i)}\right\rangle$ & $\left|\theta_{1}\right\rangle\left|\theta_{2}\right\rangle\left|\tilde{\theta}_{3}\right\rangle$ & $\pm \theta_{1} \theta_{2} \pm \theta_{1} \theta_{3} \pm \theta_{2} \theta_{3}$ & $\pm\left|\psi_{0}\right\rangle\left|\psi_{0}\right\rangle\left|\varphi_{1}\right\rangle \pm\left|\psi_{0}\right\rangle\left|\psi_{1}\right\rangle\left|\varphi_{0}\right\rangle \pm\left|\psi_{1}\right\rangle\left|\psi_{0}\right\rangle\left|\varphi_{0}\right\rangle$ \\
\hline$\left|W_{5}^{(i)}\right\rangle$ & $\left|\tilde{\theta}_{1}\right\rangle\left|\tilde{\theta}_{2}\right\rangle\left|\theta_{3}\right\rangle$ & $\pm \theta_{1} \theta_{2} \pm \theta_{1} \theta_{3} \pm \theta_{2} \theta_{3}$ & $\pm\left|\varphi_{0}\right\rangle\left|\varphi_{0}\right\rangle\left|\psi_{1}\right\rangle \pm\left|\varphi_{0}\right\rangle\left|\varphi_{1}\right\rangle\left|\psi_{0}\right\rangle \pm\left|\varphi_{1}\right\rangle\left|\varphi_{0}\right\rangle\left|\psi_{0}\right\rangle$ \\
\hline$\left|W_{6}^{(i)}\right\rangle$ & $\left|\tilde{\theta}_{1}\right\rangle\left|\theta_{2}\right\rangle\left|\tilde{\theta}_{3}\right\rangle$ & $\pm \theta_{1} \theta_{2} \pm \theta_{1} \theta_{3} \pm \theta_{2} \theta_{3}$ & $\pm\left|\varphi_{0}\right\rangle\left|\psi_{0}\right\rangle\left|\varphi_{1}\right\rangle \pm\left|\varphi_{0}\right\rangle\left|\psi_{1}\right\rangle\left|\varphi_{0}\right\rangle \pm\left|\varphi_{1}\right\rangle\left|\psi_{0}\right\rangle\left|\varphi_{0}\right\rangle$ \\
\hline$\left|W_{7}^{(i)}\right\rangle$ & $\left|\theta_{1}\right\rangle\left|\tilde{\theta}_{2}\right\rangle\left|\tilde{\theta}_{3}\right\rangle$ & $\pm \theta_{1} \theta_{2} \pm \theta_{1} \theta_{3} \pm \theta_{2} \theta_{3}$ & $\pm\left|\psi_{0}\right\rangle\left|\varphi_{0}\right\rangle\left|\varphi_{1}\right\rangle \pm\left|\psi_{0}\right\rangle\left|\varphi_{1}\right\rangle\left|\varphi_{0}\right\rangle \pm\left|\psi_{1}\right\rangle\left|\varphi_{0}\right\rangle\left|\varphi_{0}\right\rangle$ \\
\hline$\left|W_{8}^{(i)}\right\rangle$ & $\left|\tilde{\theta}_{1}\right\rangle\left|\tilde{\theta}_{2}\right\rangle\left|\tilde{\theta}_{3}\right\rangle$ & $\pm \theta_{1} \theta_{2} \pm \theta_{1} \theta_{3} \pm \theta_{2} \theta_{3}$ & $\pm\left|\varphi_{0}\right\rangle\left|\varphi_{0}\right\rangle\left|\varphi_{1}\right\rangle \pm\left|\varphi_{0}\right\rangle\left|\varphi_{1}\right\rangle\left|\varphi_{0}\right\rangle \pm\left|\varphi_{1}\right\rangle\left|\varphi_{0}\right\rangle\left|\varphi_{0}\right\rangle$ \\
\hline$\left|W_{1}^{\prime}\right\rangle$ & $|\theta\rangle|\theta\rangle|\theta\rangle$ & 1 & $-\left|\psi_{0}\right\rangle\left|\psi_{0}\right\rangle\left|\psi_{1}\right\rangle+\left|\psi_{0}\right\rangle\left|\psi_{1}\right\rangle\left|\psi_{0}\right\rangle-\left|\psi_{1}\right\rangle\left|\psi_{0}\right\rangle\left|\psi_{0}\right\rangle$ \\
\hline$\left|W_{2}^{\prime}\right\rangle$ & $|\tilde{\theta}\rangle|\theta\rangle|\theta\rangle$ & 1 & $-\left|\varphi_{0}\right\rangle\left|\psi_{0}\right\rangle\left|\psi_{1}\right\rangle+\left|\varphi_{0}\right\rangle\left|\psi_{1}\right\rangle\left|\psi_{0}\right\rangle-\left|\varphi_{1}\right\rangle\left|\psi_{0}\right\rangle\left|\psi_{0}\right\rangle$ \\
\hline$\left|W_{3}^{\prime}\right\rangle$ & $|\theta\rangle|\tilde{\theta}\rangle|\theta\rangle$ & 1 & $-\left|\psi_{0}\right\rangle\left|\varphi_{0}\right\rangle\left|\psi_{1}\right\rangle+\left|\psi_{0}\right\rangle\left|\varphi_{1}\right\rangle\left|\psi_{0}\right\rangle-\left|\psi_{1}\right\rangle\left|\varphi_{0}\right\rangle\left|\psi_{0}\right\rangle$ \\
\hline$\left|W_{4}^{\prime}\right\rangle$ & $|\theta\rangle|\theta\rangle|\tilde{\sigma}\rangle$ & 1 & $-\left|\psi_{0}\right\rangle\left|\psi_{0}\right\rangle\left|\varphi_{1}\right\rangle+\left|\psi_{0}\right\rangle\left|\psi_{1}\right\rangle\left|\varphi_{0}\right\rangle-\left|\psi_{1}\right\rangle\left|\psi_{0}\right\rangle\left|\varphi_{0}\right\rangle$ \\
\hline$\left|W_{5}^{\prime}\right\rangle$ & $|\tilde{\theta}\rangle|\tilde{\theta}\rangle|\theta\rangle$ & 1 & $-\left|\varphi_{0}\right\rangle\left|\varphi_{0}\right\rangle\left|\psi_{1}\right\rangle+\left|\varphi_{0}\right\rangle\left|\varphi_{1}\right\rangle\left|\psi_{0}\right\rangle-\left|\varphi_{1}\right\rangle\left|\varphi_{0}\right\rangle\left|\psi_{0}\right\rangle$ \\
\hline$\left|W_{6}^{\prime}\right\rangle$ & $|\tilde{\theta}\rangle|\theta\rangle|\tilde{\sigma}\rangle$ & 1 & $-\left|\varphi_{0}\right\rangle\left|\psi_{0}\right\rangle\left|\varphi_{1}\right\rangle+\left|\varphi_{0}\right\rangle\left|\psi_{1}\right\rangle\left|\varphi_{0}\right\rangle-\left|\varphi_{1}\right\rangle\left|\psi_{0}\right\rangle\left|\varphi_{0}\right\rangle$ \\
\hline$\left|W_{7}^{\prime}\right\rangle$ & $|\theta\rangle|\tilde{\theta}\rangle|\tilde{\sigma}\rangle$ & 1 & $-\left|\psi_{0}\right\rangle\left|\varphi_{0}\right\rangle\left|\varphi_{1}\right\rangle+\left|\psi_{0}\right\rangle\left|\varphi_{1}\right\rangle\left|\varphi_{0}\right\rangle-\left|\psi_{1}\right\rangle\left|\varphi_{0}\right\rangle\left|\varphi_{0}\right\rangle$ \\
\hline$\left|W_{8}^{\prime}\right\rangle$ & $|\tilde{\theta}\rangle|\tilde{\theta}\rangle|\tilde{\theta}\rangle$ & 1 & $-\left|\varphi_{0}\right\rangle\left|\varphi_{0}\right\rangle\left|\varphi_{1}\right\rangle+\left|\varphi_{0}\right\rangle\left|\varphi_{1}\right\rangle\left|\varphi_{0}\right\rangle-\left|\varphi_{1}\right\rangle\left|\varphi_{0}\right\rangle\left|\varphi_{0}\right\rangle$ \\
\hline
\end{tabular}
$\theta_{1} \theta_{3}+\theta_{2} \theta_{3}, \theta_{1} \theta_{2}+\theta_{1} \theta_{3}-\theta_{2} \theta_{3}, \ldots,-\theta_{1} \theta_{2}-\theta_{1} \theta_{3}-\theta_{2} \theta_{3}$.

4.3. Бисепарабельные псевдосостояния. В данном пункте мы используем ФПЭКС для построения бисепарабельных псевдосостояний. В зависимости от того, как разделено на две части заданное состояние, существует частичная запутанность подсистем. Например, если чистое состояние $|A B C\rangle$ включает в себя три подсистемы $A, B$ и $C$, одна из подсистем, скажем, $A$, может быть отделена, а две другие, $B$ и $C$, запутанны. 
Рассмотрим для иллюстрации следующие примеры:

$$
\begin{aligned}
& \int d \theta_{1} d \theta_{2} d \theta_{3}\left(\theta_{1} \theta_{2} \pm \theta_{1} \theta_{3}\right)\left|\theta_{1}\right\rangle\left|\theta_{2}\right\rangle\left|\theta_{3}\right\rangle=\left|\psi_{0}\right\rangle_{(1)} \otimes\left|B_{1}^{ \pm}\right\rangle_{(2,3)} \\
& \int d \theta_{1} d \theta_{2} d \theta_{3}\left(\theta_{3} \theta_{2} \theta_{1} \mp \theta_{1}\right)\left|\theta_{1}\right\rangle\left|\theta_{2}\right\rangle\left|\theta_{3}\right\rangle=\left|\psi_{0}\right\rangle_{(1)} \otimes\left|B_{1}^{\prime \pm}\right\rangle_{(2,3)} \\
& \int d \theta_{1} d \theta_{2} d \theta_{3}\left(\theta_{1} \theta_{2} \mp \theta_{3} \theta_{2}\right)\left|\theta_{1}\right\rangle\left|\theta_{2}\right\rangle\left|\theta_{3}\right\rangle=\left|\psi_{0}\right\rangle_{(2)} \otimes\left|B_{1}^{ \pm}\right\rangle_{(1,3)} .
\end{aligned}
$$

Два первых примера показывают, что подсистема $(2,3)$ является псевдосостоянием Белла, отделенным от подсистемы 1 ; в последнем примере то же относится к подсистемам $(1,3)$ и 2 . Как показывают эти примеры, можно найти различные бисепарабельные состояния, просто рассматривая интегрирование по тензорному произведению $\left|\theta_{1}\right\rangle\left|\theta_{2}\right\rangle\left|\theta_{3}\right\rangle$ с различными весовыми функциями. Однако следует заметить, что состояния из семейства $W^{\prime}$ не дают никаких бисепарабельных состояний.

\section{5. ЗАПУТАННОСТЬ МНОГОЧАСТИЧНЫХ ПСЕВДОЭРМИТОВЫХ СОСТОЯНИЙ}

В данном разделе мы изучаем запутанность псевдосостояний Белла, используя функцию конкуренции, и запутанность GHZ- и W-псевдосостояний, используя среднюю энтропию. С этой целью рассмотрим следующие двухуровневые псевдоэрмитовы гамильтонианы:

$$
H_{i}=\left(\begin{array}{cc}
r_{i} e^{i \beta_{i}} & s_{i} \\
t_{i} & r_{i} e^{-i \beta_{i}}
\end{array}\right), \quad i=1,2,3
$$

где индекс $i$ отвечает $i$-й системе, а $r_{i}, s_{i}, t_{i}$ и $\beta_{i}$ - действительные числа. Такой гамильтониан неэрмитов, хотя его собственные значения действительны, если выполнено неравенство $s_{i} t_{i}>r_{i}^{2} \sin ^{2} \beta_{i}$ [48]. Предположим, что системы живут в четырех- и восьмимерных гильбертовых пространствах и их динамика управляется соответственно гамильтонианами $H_{1} \otimes H_{2}$ и $H_{1} \otimes H_{2} \otimes H_{3}$. Биортонормированные собственные состояния гамильтонианов $H_{i}$ и $H_{i}^{\dagger}$ таковы:

$$
\begin{array}{rlrl}
\left|\psi_{0}\right\rangle^{(i)} & =\frac{1}{\sqrt{2} \cos \alpha_{i}}\left(\begin{array}{c}
e^{i \alpha_{i} / 2} \\
e^{-i \alpha_{i} / 2}
\end{array}\right), & \left|\varphi_{0}\right\rangle^{(i)}=\frac{1}{\sqrt{2} \cos \alpha_{i}}\left(\begin{array}{c}
e^{-i \alpha_{i} / 2} \\
e^{i \alpha_{i} / 2}
\end{array}\right), \\
\left|\psi_{1}\right\rangle^{(i)}=\frac{1}{\sqrt{2} \cos \alpha_{i}}\left(\begin{array}{c}
e^{-i \alpha_{i} / 2} \\
-e^{i \alpha_{i} / 2}
\end{array}\right), & \left|\varphi_{1}\right\rangle^{(i)}=\frac{1}{\sqrt{2} \cos \alpha_{i}}\left(\begin{array}{c}
e^{i \alpha_{i} / 2} \\
-e^{-i \alpha_{i} / 2}
\end{array}\right),
\end{array}
$$

где $\sin \alpha_{i}=\left(r_{i} / \sqrt{s_{i} t_{i}}\right) \sin \beta_{i}$. Матрица $\eta=\sum_{i}\left|\varphi_{i}\right\rangle\left\langle\varphi_{i}\right|$ (псевдо)метрического оператора для гамильтониана $H_{i}$ имеет вид

$$
\eta=\frac{1}{\cos ^{2} \alpha_{i}}\left(\begin{array}{cc}
1 & -i \sin \alpha_{i} \\
i \sin \alpha_{i} & 1
\end{array}\right) .
$$

В следующем пункте мы рассматриваем псевдосостояния Белла. 


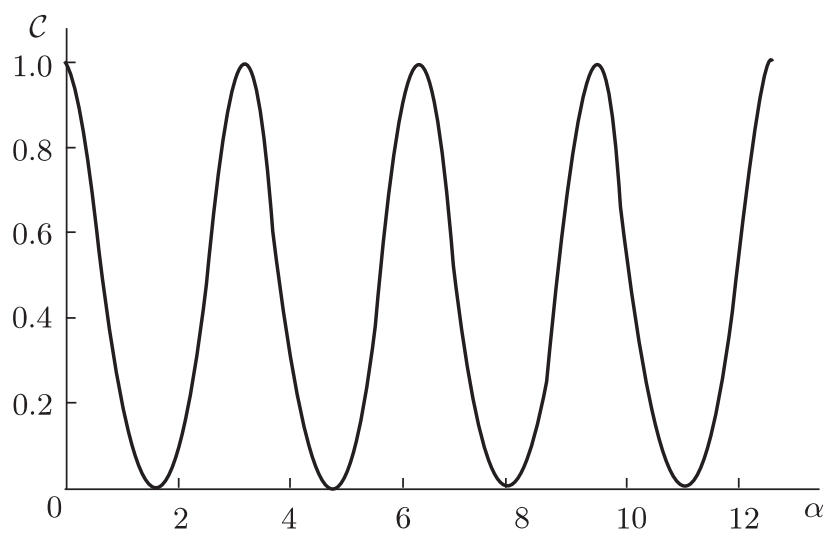

Рис. 1. Конкуренция состояний $\left|B_{2}^{-}\right\rangle$и $\left|B_{3}^{-}\right\rangle$как функция от $\alpha$.

5.1. Запутанность псевдосостояний Белла. Хорошо известно, что запутанность двухкубитного состояния $|\psi\rangle$ можно выразить с помощью функции конкуренции [49], [50]

$$
\mathcal{C}(|\psi\rangle) \equiv\left|\left\langle\psi\left|\sigma_{y} \otimes \sigma_{y}\right| \psi^{*}\right\rangle\right|
$$

где $\sigma_{y}$ есть $y$-матрица Паули $\left(\begin{array}{rr}0 & -i \\ i & 0\end{array}\right)$ и $\left|\psi^{*}\right\rangle$ - состояние, комплексно-сопряженное к $|\psi\rangle$. Используем функцию конкуренции, чтобы количественно описать запутанность псевдосостояний Белла.

После нормировки псевдосостояний Белла из табл. 1 и подстановки явного вида состояний $\left|\psi_{k}\right\rangle^{(i)}$ и $\left|\varphi_{k}\right\rangle^{(i)}(k=0,1)$ из $(5.2)$ соответствующие конкуренции принимают вид

$$
\begin{aligned}
& \mathcal{C}\left(\left|B_{1}^{-}\right\rangle\right)=\mathcal{C}\left(\left|B_{4}^{-}\right\rangle\right)=\left|\frac{\cos \alpha_{1} \cos \alpha_{2}}{1-\sin \alpha_{1} \sin \alpha_{2}}\right| \\
& \mathcal{C}\left(\left|B_{2}^{-}\right\rangle\right)=\mathcal{C}\left(\left|B_{3}^{-}\right\rangle\right)=\left|\frac{\cos \alpha_{1} \cos \alpha_{2}}{1+\sin \alpha_{1} \sin \alpha_{2}}\right|
\end{aligned}
$$

Мы сосредоточили свое внимание на нижней трети таблицы 1 , аналогичные рассуждения можно провести и для остальных псевдосостояний Белла. Таким образом, конкуренция состояний $\left|B_{j}^{-}\right\rangle$есть периодическая функция по параметрам $\alpha_{1}$ и $\alpha_{2}$ с периодом $\pi$, т. е. $\mathcal{C}\left(\alpha_{1}, \alpha_{2}\right)=\mathcal{C}\left(\alpha_{1}+m \pi, \alpha_{2}+m \pi\right)$, где $m \in \mathbb{Z}$. Из формул (5.5) видно, что в обоих случаях $\mathcal{C}_{\max }=1$ и $\mathcal{C}_{\min }=0$, и эти максимальное и минимальное значения отвечают $\alpha_{1}=\alpha_{2}=m \pi$ и $\alpha_{1}=\alpha_{2}=(2 m+1) \pi / 2$. В специальном случае $\alpha_{1}=\alpha_{2}=\alpha$ соотношения (5.5) принимают вид

$$
\mathcal{C}\left(\left|B_{1}^{-}\right\rangle\right)=\mathcal{C}\left(\left|B_{4}^{-}\right\rangle\right)=1, \quad \mathcal{C}\left(\left|B_{2}^{-}\right\rangle\right)=\mathcal{C}\left(\left|B_{3}^{-}\right\rangle\right)=\frac{\cos ^{2} \alpha}{1+\sin ^{2} \alpha}
$$

Мы получили, что $\mathcal{C}$ для $\left|B_{1}^{-}\right\rangle$и $\left|B_{4}^{-}\right\rangle$не зависит от $\alpha$, что не является удивительным, поскольку при $\alpha_{1}=\alpha_{2}=\alpha$ состояния сводятся к обычному состоянию Белла $\left|\Psi^{-}\right\rangle$ 
с точностью до фазового множителя $e^{-i \pi}$,

$$
\begin{gathered}
\left|B_{1}\right\rangle^{-}=\frac{\left|\psi_{0}\right\rangle\left|\psi_{1}\right\rangle-\left|\psi_{1}\right\rangle\left|\psi_{0}\right\rangle}{\|\left|B_{1}^{-}\right\rangle \|}=-\frac{|01\rangle-|10\rangle}{\sqrt{2}}, \\
\left|B_{4}\right\rangle^{-}=\frac{\left|\varphi_{0}\right\rangle\left|\varphi_{1}\right\rangle-\left|\varphi_{1}\right\rangle\left|\varphi_{0}\right\rangle}{\|\left|B_{4}^{-}\right\rangle \|}=-\frac{|01\rangle-|10\rangle}{\sqrt{2}} .
\end{gathered}
$$

Напротив, конкуренция состояний $\left|B_{2}^{-}\right\rangle$и $\left|B_{3}^{-}\right\rangle$зависит от $\alpha$ (см. рис. 1).

Простое вычисление показывает, что при $\alpha_{1}=\alpha_{2}=\alpha$ следующие псевдосостояния Белла сводятся к стандартным состояниям Белла:

$$
\left|B_{2}^{\prime-}\right\rangle=\left|B_{3}^{\prime-}\right\rangle=\left|\Psi^{+}\right\rangle, \quad\left|B_{1}^{\prime+}\right\rangle=\left|B_{4}^{\prime+}\right\rangle=\left|\Phi^{+}\right\rangle, \quad\left|B_{2}^{+}\right\rangle=\left|B_{3}^{+}\right\rangle=\left|\Phi^{-}\right\rangle .
$$

Рассмотрим два частных случая, представляющих интерес с точки зрения распада, вызванного дипольным взаимодействием.

1. Если $s t=r^{2} \sin ^{2} \beta$, то $\mathcal{C}\left(\left|B_{2}^{-}\right\rangle\right)=\mathcal{C}\left(\left|B_{3}^{-}\right\rangle\right)=0$.

2. Если $r=\delta / 2, \beta=-\pi / 2, t=s$, то гамильтониан сводится к

$$
H_{1,2}=\frac{1}{2}\left(\begin{array}{cc}
-i \delta & 2 s \\
2 s & i \delta
\end{array}\right) .
$$

Такой гамильтониан возникает при взаимодействии двухуровневого атома с электромагнитным полем, при этом действительное число $\delta$ есть скорость распада для высшего и низшего уровня, а величина $s$ описывает межуровневый элемент матрицы взаимодействия излучение-атом, записанный в картине взаимодействия с приближением вращающейся волны [25], [51], [52]. В этом случае конкуренция как функция от $s$ и $\delta$ имеет вид

$$
\mathcal{C}\left(\left|B_{2}^{-}\right\rangle\right)=\mathcal{C}\left(\left|B_{3}^{-}\right\rangle\right)=\frac{4 s^{2}-\delta^{2}}{4 s^{2}+\delta^{2}} .
$$

Поскольку $\sin \alpha=-\delta / 2 s$, мы имеем $4 s^{2}-\delta^{2} \geqslant 0$, что гарантирует неотрицательность конкуренции. Рис. 2 показывает, как ведет себя конкуренция (5.10) в диапазоне значений $1 \leqslant s \leqslant 2$ и $-2 \leqslant \delta \leqslant 2$.

5.2. Запутанность GHZ-псевдосостояний и $\mathrm{W}$-псевдосостояний. В качестве следующего шага мы дадим количественное описание запутанности GHZи $\mathrm{W}$-псевдосостояний. Рассмотрим среднюю энтропию $\left\langle S_{\mathrm{L}}\right\rangle$, являющуюся хорошей мерой для описания запутанности,

$$
\left\langle S_{\mathrm{L}}\right\rangle=\left(\begin{array}{c}
N \\
n
\end{array}\right)^{-1} \sum_{A_{n}} S_{\mathrm{L}}^{\left(A_{n} ; B_{N-n}\right)} ;
$$

мы определили ее через линейную энтропию [53]

$$
S_{\mathrm{L}}^{\left(A_{n} ; B_{N-n}\right)}=\frac{d}{d-1}\left(1-\operatorname{Tr}_{A_{n}}\left[\rho_{A_{n}}\right]^{2}\right), \quad \rho_{A_{n}}=\operatorname{Tr}_{B_{N-n}}[\rho],
$$

где $d=\min \left\{2^{n}, 2^{N-n}\right\}$ есть размерность редуцированной матрицы плотности $\rho_{A_{n}}$. Следует отметить, что, хотя линейная энтропия и энтропия фон Неймана [54] являются схожими мерами смешанности состояний, линейную энтропию вычислить 


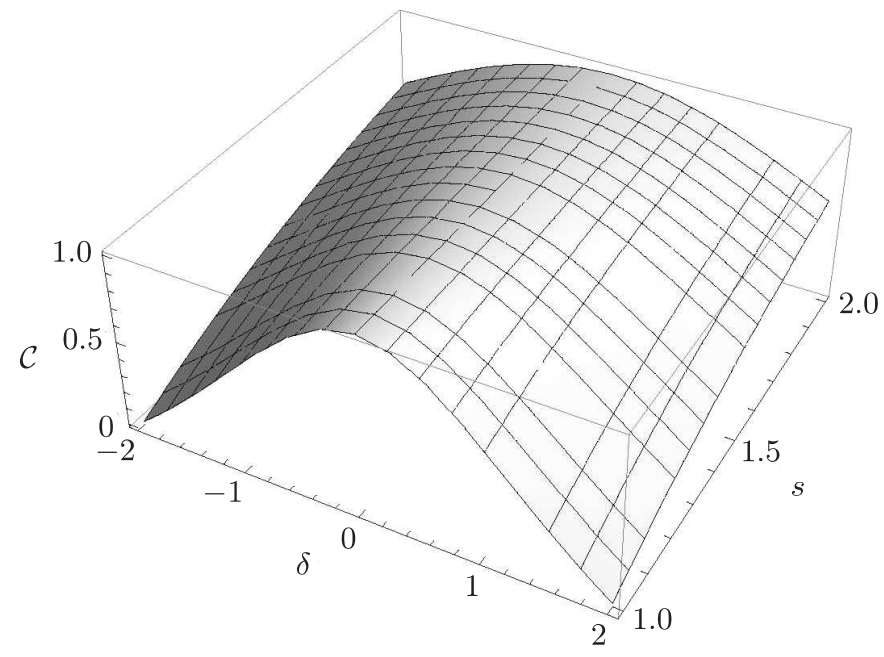

Рис. 2. Конкуренция состояний $\left|B_{2}^{-}\right\rangle$и $\left|B_{3}^{-}\right\rangle$как функция от $\delta$ и $s$; в точ$\operatorname{kax}(\delta, s)$ с $\delta=0$ конкуренция равна единице, состояния максимально запутанны.

проще, поскольку при этом не требуется диагонализация матрицы плотности. Линейная энтропия меняется в диапазоне от нуля (полностью чистое состояние) до единицы (полностью смешанное состояние).

Опираясь на меру запутанности, заданную как средняя энтропия, в качестве примера исследуем запутанность нормированных состояний $\left|G_{1}^{+}\right\rangle,\left|\mathcal{W}_{7}^{(+,+,+)}\right\rangle$и $\left|\mathcal{W}_{6}^{(-,+,-)}\right\rangle$ (последние мы для простоты обозначим как $W_{7}$ и $W_{6}$ ). Имеем

$$
\begin{aligned}
\left|G_{1}^{+}\right\rangle & =\frac{\left|\varphi_{0}\right\rangle\left|\varphi_{0}\right\rangle\left|\varphi_{0}\right\rangle \pm\left|\varphi_{1}\right\rangle\left|\varphi_{1}\right\rangle\left|\varphi_{1}\right\rangle}{\|\left|G_{1}^{+}\right\rangle \|}, \\
\left|\mathcal{W}_{7}\right\rangle & =\frac{\left|\psi_{0}\right\rangle\left|\varphi_{0}\right\rangle\left|\varphi_{1}\right\rangle+\left|\psi_{0}\right\rangle\left|\varphi_{1}\right\rangle\left|\varphi_{0}\right\rangle+\left|\psi_{1}\right\rangle\left|\varphi_{0}\right\rangle\left|\varphi_{0}\right\rangle}{\|\left|W_{7}\right\rangle \|} \\
\left|\mathcal{W}_{6}\right\rangle & =\frac{-\left|\varphi_{0}\right\rangle\left|\psi_{0}\right\rangle\left|\varphi_{1}\right\rangle+\left|\varphi_{0}\right\rangle\left|\psi_{1}\right\rangle\left|\varphi_{0}\right\rangle-\left|\varphi_{1}\right\rangle\left|\psi_{0}\right\rangle\left|\varphi_{0}\right\rangle}{\|\left|W_{6}\right\rangle \|} .
\end{aligned}
$$

В соответствии с определением (5.11) средняя энтропия нормированного состояния $\left|G_{1}^{+}\right\rangle$задается как

$$
\left\langle S_{\mathrm{L}}\right\rangle_{\left(G_{1}^{+}\right)}=\frac{5+\cos 2 \alpha_{2}-2 \sin ^{2} \alpha_{1}\left(1+\cos ^{2} \alpha_{3} \sin ^{2} \alpha_{2}\right)+\left(\cos 2 \alpha_{1} \cos 2 \alpha_{2}-3\right) \sin ^{2} \alpha_{3}}{6} .
$$

Прямое вычисление показывает, что средняя энтропия остальных GHZ-псевдосостояний такая же.

Как и выше, рассмотрим квантовые состояния с $\alpha_{1}=\alpha_{2}=\alpha_{3}=\alpha$, тогда

$$
\left\langle S_{\mathrm{L}}\right\rangle_{\left(G_{1}^{+}\right)}=\frac{1}{2} \cos ^{4} \alpha(3-\cos 2 \alpha) .
$$

На рис. 3 изображена средняя энтропия состояния $\left|G_{1}^{+}\right\rangle$как функция от параметра $\alpha$. Максимум и минимум средней энтропии для GHZ-псевдосостояний достигаются при $\alpha=k \pi$ и $\alpha=(2 k+1) \pi / 2$. 


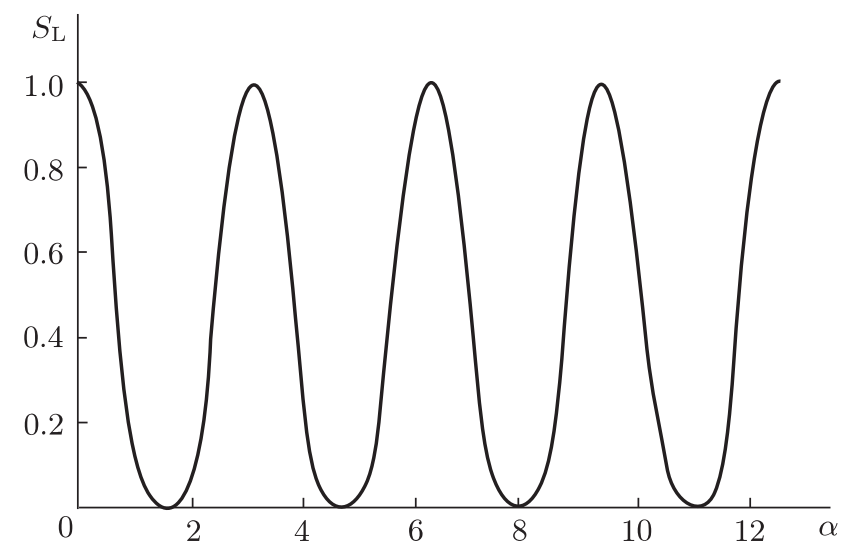

Рис. 3. Средняя энтропия всех GHZ-псевдосостояний как функция от $\alpha$.

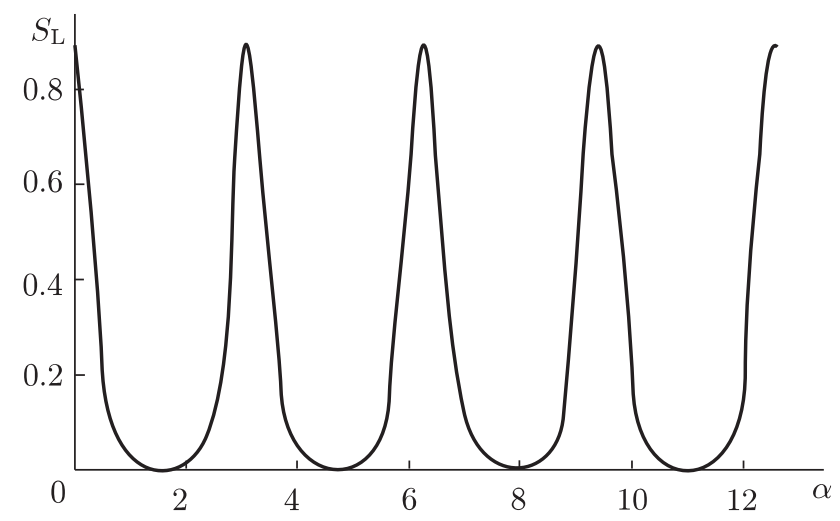

Рис. 4. Средняя энтропия состояния $\left|\mathcal{W}_{7}\right\rangle$ как функция от $\alpha$. Нижняя граница (ноль) и верхняя граница (8/9) отвечают сепарабельному и максимально запутанному псевдосостояниям $\left|\mathcal{W}_{7}\right\rangle$.

В качестве следующего примера рассмотрим нормированное состояние $\left|\mathcal{W}_{7}\right\rangle$, при этом средняя энтропия в случае различных и равных $\alpha_{i}$ равна

$$
\begin{aligned}
\left\langle S_{\mathrm{L}}\right\rangle_{\left(\mathcal{W}_{7}\right)=} & \frac{1}{3\left(2 \sin \alpha_{2} \sin \alpha_{3}-2 \sin \alpha_{1}\left(\sin \alpha_{2}+\sin \alpha_{3}\right)+3\right)^{2}} \times \\
& \times\left(2\left(\cos 2 \alpha_{1}+\cos 2 \alpha_{2}+2\right) \cos 2 \alpha_{3}+\cos 2\left(\alpha_{1}-\alpha_{2}\right)+\right. \\
& \left.+\cos 2\left(\alpha_{1}+\alpha_{2}\right)+4 \cos 2 \alpha_{1}+4 \cos 2 \alpha_{2}+6\right), \\
\left\langle S_{\mathrm{L}}\right\rangle_{\left(\mathcal{W}_{7}\right)}= & \frac{8 \cos ^{4} \alpha}{(\cos 2 \alpha+2)^{2}} .
\end{aligned}
$$

На рис. 4 показана средняя энтропия состояния $\left|\mathcal{W}_{7}\right\rangle$, в случае равных $\alpha_{i}$ она лежит в интервале $0 \leqslant\left\langle S_{\mathrm{L}}(\alpha)\right\rangle_{\left(\mathcal{W}_{7}\right)} \leqslant 8 / 9$, нижняя и верхняя граница достигаются при $\alpha=k \pi$ и $\alpha_{k}=(2 k+1) \pi / 2$. 


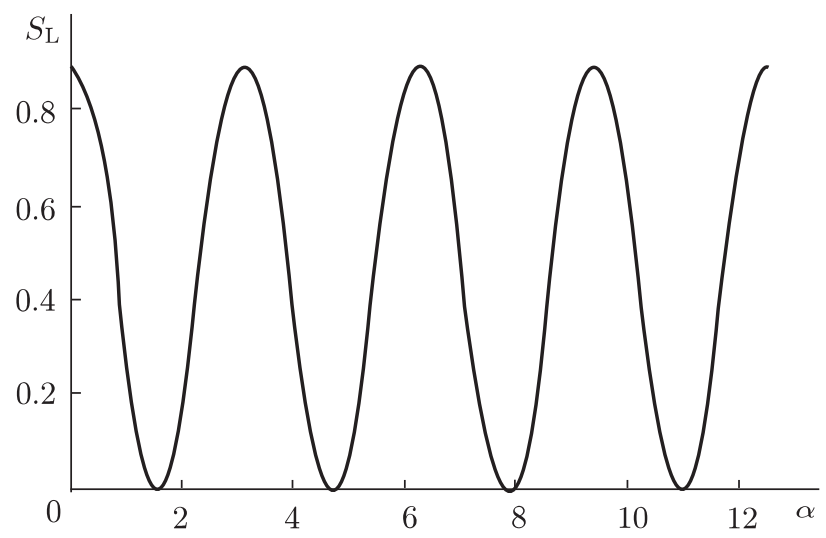

Рис. 5. Средняя энтропия состояния $\left|W_{6}\right\rangle$ как функция от $\alpha$.

В качестве последнего примера рассмотрим среднюю энтропию состояния $\left|\mathcal{W}_{6}\right\rangle$. С учетом определения (5.11) мы выводим следующее выражение:

$$
\begin{aligned}
\left\langle S_{\mathrm{L}}\right\rangle_{\left(\mathcal{W}_{6}\right)}= & \frac{1}{3\left(2 \sin \alpha_{2} \sin \alpha_{3}+2 \sin \alpha_{1}\left(\sin \alpha_{2}+\sin \alpha_{3}\right)+3\right)^{2}} \times \\
& \times\left(2\left(\cos 2 \alpha_{1}+\cos 2 \alpha_{2}+2\right) \cos 2 \alpha_{3}+\cos 2\left(\alpha_{1}-\alpha_{2}\right)+\right. \\
& \left.\quad+\cos 2\left(\alpha_{1}+\alpha_{2}\right)+4 \cos 2 \alpha_{1}+4 \cos 2 \alpha_{2}+6\right) .
\end{aligned}
$$

Нетрудно проверить, что в случае $\alpha_{1}=\alpha_{2}=\alpha_{3}=\alpha$ это выражение сводится $\mathrm{k}$

$$
\left\langle S_{\mathrm{L}}\right\rangle_{\left(\mathcal{W}_{6}\right)}=\frac{8 \cos ^{4} \alpha}{9(\cos 2 \alpha-2)^{2}} .
$$

На рис. 5 изображена средняя энтропия состояния $\left|\mathcal{W}_{6}\right\rangle$ как функция от параметра $\alpha$. Как и в предыдущем случае, максимум средней энтропии в точности равен максимуму средней энтропии для запутанных состояний, описывающихся стандартным эрмитовым гамильтонианом.

Изложенный выше метод можно распространить на многочастичные $n$-уровневые системы.

\section{6. ЗАКЛЮЧЕНИЕ}

В представленной статье мы построили псевдоэрмитову версию хорошо известных максимально запутанных чистых состояний, таких как состояния Белла, GHZсостояния, W-состояния и бисепарабельные состояния, путем интегрирования по тензорному произведению одномодовых ФПЭКС с использованием грассмановой весовой функции. В качестве поясняющего примера мы рассмотрели в явном виде биортонормированнные собственные состояния псевдоэрмитова гамильтониана, который возникает при взаимодействии двухуровневого атома с электромагнитным полем. Для количественного описания запутанности двухкубитных состояний (псевдосостояний Белла) и трехкубитных состояний (GHZ- и W-псевдосостояний) мы использовали меры запутанности, заданные соответственно функцией конкуренции 
и средней линейной энтропией. Обнаружилось, что при $\alpha_{1}=\alpha_{2}=\alpha$ псевдосостояния Белла $\left|B_{1}^{-}\right\rangle$и $\left|B_{4}^{-}\right\rangle$с точностью до фазового множителя $e^{-i \pi}$ совпадают со стандартным состоянием Белла $\left|\Psi^{-}\right\rangle$. Аналогично состояния $\left|B_{2}^{\prime-}\right\rangle$ и $\left|B_{3}^{\prime-}\right\rangle$ совпадают с $\left|\Psi^{-}\right\rangle$, состояния $\left|B_{1}^{\prime+}\right\rangle$ и $\left|B_{4}^{\prime+}\right\rangle$ совпадают с $\left|\Phi^{+}\right\rangle$, а состояния $\left|B_{2}^{+}\right\rangle$и $\left|B_{3}^{+}\right\rangle$ сводятся к $\left|\Phi^{-}\right\rangle$.

\section{Список литературы}

[1] M. A. Nielsen, I. L. Chuang, Quantum Computation and Quantum Information, Cambridge Univ. Press, Cambridge, 2000.

[2] O. Gühne, G. Tóth, "Entanglement detection", Phys. Rep., 474:1-6 (2009), 1-75.

[3] S. J. van Enk, N. Lütkenhaus, H. J. Kimble, "Experimental procedures for entanglement verification", Phys. Rev. A, 75:5 (2007), 052318, 14 pp.

[4] P. Horodecki, "Measuring quantum entanglement without prior state reconstruction", Phys. Rev. Lett., 90:16 (2003), 167901, 4 pp.

[5] P. Horodecki, A. Ekert, "Method for direct detection of quantum entanglement", Phys. Rev. Lett., 89:12 (2002), 127902, 4 pp.

[6] S. P. Walborn, P. H. Souto Ribeiro, L. Davidovich, F. Mintert, A. Buchleitner, "Experimental determination of entanglement with a single measurement", Nature, 440:7087 (2006), $1022-1024$.

[7] C. Schmid, N. Kiesel, W. Wieczorek, H. Weinfurter, "Experimental direct observation of mixed state entanglement", Phys. Rev. Lett., 101:26 (2008), 260505, 4 pp.

[8] G. Vidal, "Entanglement monotones", J. Modern Opt., 47:2-3 (2000), 355-376.

[9] J.S. Bell, Speakable and Unspeakable in Quantum Mechanics, Cambridge Univ. Press, Cambridge, 1987.

[10] S. Lloyd, S. L. Braunstein, "Quantum computation over continuous variables", Phys. Rev. Lett., 82:8 (1999), 1784-1787.

[11] S. J. van Enk, "Decoherence of multidimensional entangled coherent states", Phys. Rev. A, 72:2 (2005), 022308, 6 pp.

[12] S. J. van Enk, O. Hirota, "Entangled coherent states: teleportation and decoherence", Phys. Rev. A, 64:2 (2001), 022313, 6 pp.

[13] H. Fu, X. Wang, A. I. Solomon, "Maximal entanglement of nonorthogonal states: classification", Phys. Lett. A, 291:2-3 (2001), 73-76.

[14] X. Wang, B.C. Sanders, "Multipartite entangled coherent states", Phys. Rev. A, 65:1 (2002), 012303, 7 pp.

[15] X. Wang, "Bipartite entangled non-orthogonal states", J. Phys. A: Math. Gen., 35:1 (2002), $165-174$.

[16] X. Wang, B. C. Sanders, S. H. Pan, "Entangled coherent states for systems with $S U(2)$ and SU (1,1) symmetries", J. Phys. A: Math. Gen., 33:41 (2000), 7451-7467.

[17] X. Wang, "Entanglement in the quantum Heisenberg XY model", Phys. Rev. A, 64:1 (2001), 012313, 7 pp.

[18] L. Borsten, D. Dahanayake, M. J. Duff, W. Rubens, "Superqubits", Phys. Rev. D, 81:10 (2010), 105023, 16 pp.

[19] F. C. Khanna, J. M. C. Malbouisson, A. E. Santana, E. S. Santos, "Maximum entanglement in squeezed boson and fermion states", Phys. Rev. A, 76:2 (2007), 022109, 5 pp.

[20] G. Najarbashi, Y. Maleki, "Entanglement of Grassmannian coherent states for multi-partite n-level systems", SIGMA, 7 (2011), 011, 11 pp., arXiv: 1008.4836.

[21] G. Najarbashi, Y. Maleki, "Maximal entanglement of two-qubit states constructed by linearly independent coherent states", Internat. J. Theor. Phys., 50:8 (2011), 2601-2608.

[22] Y. Maleki, "Para-Grassmannian coherent and squeezed states for pseudo-Hermitian q-oscillator and their entanglement", SIGMA, 7 (2011), 084, 20 pp., arXiv: 1108.5005. 
[23] S. Majid, "Random walk and the heat equation on superspace and anyspace", J. Math. Phys., 35:7 (1994), 3753-3760.

[24] D. C. Cabra, E. F. Moreno, A. Tanasă, "Para-Grassmann variables and coherent states", SIGMA, 2 (2006), 087, 8 pp.

[25] O. Cherbal, M. Drir, M. Maamache, D. A. Trifonov, "Fermionic coherent states for pseudo-Hermitian two-level systems", J. Phys. A: Math. Theor., 40:8 (2007), 1835-1844.

[26] G. Najarbashi, M. A. Fasihi, H. Fakhri, "Generalized Grassmannian coherent states for pseudo-Hermitian n-level systems", J. Phys. A: Math. Theor., 43:32 (2010), 325301, 10 pp.

[27] F. G. Scholtz, H. B. Geyer, F. J. W. Hahne, "Quasi-Hermitian operators in quantum mechanics and the variational principle", Ann. Phys., 213:1 (1992), 74-101.

[28] C. M. Bender, S. Boettcher, "Real spectra in non-Hermitian Hamiltonians having $\mathscr{P} \mathscr{T}$ symmetry", Phys. Rev. Lett., 80:24 (1998), 5243-5246.

[29] C. M. Bender, S. Boettcher, P. N. Meisenger, "PT-symmetric quantum mechanics", J. Math. Phys., 40:5 (1999), 2201-2229.

[30] C. M. Bender, G. V. Dunne, "Large-order perturbation theory for a non-Hermitian PT-symmetric Hamiltonian", J. Math. Phys., 40:10 (1999), 4616-4621.

[31] F. Cannata, G. Junker, J. Trost, "Schrödinger operators with complex potential but real spectrum", Phys. Lett. A, 246:3-4 (1998), 219-226.

[32] M. Znojil, F. Cannata, B. Bagchi, R. Roychoudhury, "Supersymmetry without Hermiticity within PT symmetric quantum mechanics", Phys. Lett. B, 483:1-3 (2000), 284-289.

[33] A. Mostafazadeh, "Pseudo-Hermiticity versus PT symmetry: the necessary condition for the reality of the spectrum of a non-Hermitian Hamiltonian", J. Math. Phys., 43:1 (2002), 205-214.

[34] A. Mostafazadeh, "Pseudo-Hermiticity versus PT-symmetry. II. A complete characterization of non-Hermitian Hamiltonians with a real spectrum", J. Math. Phys., 43:5 (2002), $2814-2816$.

[35] A. Mostafazadeh, "Pseudo-Hermitian representation of quantum mechanics", Intenat. J. Geom. Meth. Modern Phys., 7:7 (2010), 1191-1306.

[36] N. Hatano, D. R. Nelson, "Localization transitions in non-Hermitian quantum mechanics", Phys. Rev. Lett., 77:3 (1996), 570-573.

[37] N. Hatano, D. R. Nelson, "Vortex pinning and non-Hermitian quantum mechanics", Phys. Rev. B, 56:14 (1997), 8651-8673.

[38] C. H. Bennett, G. Brassard, C. Crepeau, R. Jozsa, A. Peres, W. K. Wooters, "Teleporting an unknown quantum state via dual classical and Einstein-Podolsky-Rosen channels", Phys. Rev. Lett., 70:13 (1993), 1895-1899.

[39] D. Bouwmeester, A. K. Ekert, A. Zeilinger (eds.), The Physics of Quantum Information: Quantum Cryptography, Quantum Teleportation, Quantum Computation, Springer, 2001.

[40] A. K. Pati, "Minimum classical bit for remote preparation and measurement of a qubit", Phys. Rev. A, 63:1 (2001), 014302, 3 pp.

[41] A. V. Sergienko (ed.), Quantum Communications and Cryptography, CRC Press Taylor and Francis Group, Boca Raton, FL, 2006.

[42] P. G. O. Anicich, H. Grinberg, "Grassmann coherent states for spin systems", J. Molec. Struct., 621:1-2 (2003), 9-18.

[43] S. Abe, "Adiabatic holonomy and evolution of fermionic coherent state", Phys. Rev. D, 39:8 (1989), 2327-2331.

[44] J. Ohnuki, T. Kashiwa, "Coherent states of Fermi operators and the path integral", Prog. Theor. Phys., 60:2 (1978), 548-564.

[45] Ф.А. Березин, Метод вторичного квантования, Физматлит, М., 1965.

[46] K. E. Cahill, R. J. Glauber, "Density operators for fermions", Phys. Rev. A, 59:2 (1999), $1538-1555$. 
[47] A. Acin, D. Bruß, M. Lewenstein, A. Sanpera, "Classification of mixed three-qubit states", Phys. Rev. Lett., 87:4 (2001), 040401, 4 pp.

[48] A. K. Pati, "Entanglement in non-Hermitian quantum theory", Pramana, 73:3 (2010), 485-498.

[49] W. K. Wootters, "Entanglement of formation of an arbitrary state of two qubits", Phys. Rev. Lett., 80:10 (1998), 2245-2248.

[50] S. Hill, W. K. Wootters, "Entanglement of a pair of quantum bits", Phys. Rev. Lett., 78:26 (1997), 5022-5025.

[51] W.E. Lamb, R. R. Schlicher, M. O. Scully, "Matter-field interaction in atomic physics and quantum optics", Phys. Rev. A, 36:6 (1987), 2763-2772.

[52] J. C. Garrison, E. M. Wright, "Complex geometrical phases for dissipative systems", Phys. Lett. A, 128:3-4 (1988), 177-181.

[53] A. K. Rajagopal, R. W. Rendell, "Nonextensive statistical mechanics: implications to quantum information", Europhys. News, 36:6 (2005), 221-224.

[54] M. B. Plenio, V. Vedral, "Teleportation, entanglement and thermodynamics in the quantum world", Contemp. Phys., 39:6 (2001), 431-446.

Поступила в редакцию 25.09.2017, после доработки 29.01.2018 